\title{
INTRACARDIAC ACETYLCHOLINE INFUSION AND LEFT HEART DYNAMICS IN RHEUMATIC HEART DISEASE
}

\author{
BY \\ PHILIP SAMET, WILLIAM H. BERNSTEIN, AND ROBERT S. LITWAK \\ From the Cardio-Pulmonary Laboratory, Mount Sinai Hospital, Miami Beach, and the Departments of Medicine and \\ Surgery, University of Miami School of Medicine, Coral Gables, Florida, U.S.A.* \\ Received April 27, 1961
}

Acetylcholine has received much attention of late as a potential, clinically useful, dilator agent of the pulmonary vascular bed. Right heart intracardiac infusion of acetylcholine has been employed as the method of drug administration. Recognition of the various parameters that must be kept constant to permit conclusions as to pulmonary vasodilator or vasoconstrictor activity (Fritts and Cournand, 1959) has led to studies in man in which cardiac output and rate, systemic arterial pressure, and central blood volume were measured, and animal studies in which alveolar and intrapleural pressures were evaluated. Pulmonary arterial wedge pressure has been equated with left atrial pressure in these investigations. Previous studies in this laboratory have illustrated some of the problems associated with interpretation of pulmonary arterial wedge pressure (Bernstein et al., 1960). The purpose of this study is to report the effect of intracardiac right heart acetylcholine infusion upon directly measured left atrial and left ventricular pressures.

\section{Methods AND SubJeCts}

Combined right and left heart catheterization was performed in 14 patients with rheumatic heart disease and mitral and or aortic stenosis. The diagnoses are given in Table I. Acetylcholine was infused at a constant rate via the middle lumen of a triple lumen Cournand catheter or via the proximal lumen of a double lumen catheter into the outflow tract of the right ventricle or via the proximal lumen of the triple lumen catheter into the right atrium. Systemic arterial cannulation was done with a Cournand needle. After control supine right heart and systemic arterial pressure measurements and determination of cardiac output by the Fick principle before and during acetylcholine infusion, left heart catheterization was performed in the prone position by a modification of the posterior percutaneous left atrial puncture technique of Fisher (Samet et al., 1959). Left atrial puncture was accomplished with two 7 inch long 17-T needles. Left atrial pressure was measured with a $70 \mathrm{~mm}$. length of polyethylene tubing passed into the left atrium; left ventricular pressure was measured by passing a second length of polyethylene tubing into the left ventricle. Left atrial, left ventricular, and systemic arterial pressures were measured simultaneously from the same baseline at identical strain gauge sensitivities with Statham P23G gauges.

After control pressures and control mean diastolic left atrial to left ventricular gradients and control mean systolic left ventricular to systemic arterial gradients were obtained, right heart acetylcholine infusion at a constant rate was initiated; during this infusion repeat measurements were made of pulmonary and systemic arterial pressures as well as left atrial and ventriclar pressures and diastolic mitral and systolic aortic gradients.

In catheterization number $198,0.5 \mathrm{mg} . / \mathrm{min}$. acetylcholine was infused into the right atrium.

* This project was supported in part by a grant from the National Heart Institute, H-4249. 
TABLE I

Physical Characteristics and Diagnosis in 14 Patients with Rheumatic Heart Disease

\begin{tabular}{|c|c|c|c|c|c|c|}
\hline Date & Patient & $\begin{array}{l}\text { Cath. } \\
\text { No. }\end{array}$ & Sex & $\begin{array}{l}\text { Age } \\
\text { (yr.) }\end{array}$ & $\begin{array}{l}\text { B.S.A. } \\
\left(\mathrm{m} .{ }^{2}\right)\end{array}$ & Diagnosis \\
\hline $4 / 28 / 58$ & R. Cos. & 198 & $\mathbf{M}$ & 35 & $1 \cdot 80$ & Rh.H.D., E.H., M.S., M.I., A.F., III-C \\
\hline $6 / 17 / 58$ & M. Win. & 211 & $\mathrm{~F}$ & 40 & $1 \cdot 42$ & Rh.H.D., E.H., M.S., N.S.R., III-D \\
\hline $7 / 11 / 58$ & C. Bro. & 219 & $\mathrm{~F}$ & 53 & $1 \cdot 86$ & $\begin{array}{l}\text { Rh.H.D., E.H., A.S., M.S., Minimal A.I., N.S.R. } \\
\text { II-C, } 6 \text { mos. post op. }\end{array}$ \\
\hline $11 / 17 / 58$ & L. McGo. & 251 & $\mathrm{~F}$ & 35 & $1 \cdot 46$ & Rh.H.D., E.H., M.S., T.S., N.S.R., II-C \\
\hline $1 / 5 / 59$ & B. Ruc. & 263 & M & 42 & $1 \cdot 82$ & Rh.H.D., E.H., M.S., Minimal A.I., N.S.R., II-C \\
\hline $3 / 7 / 59$ & M. Ham. & 280 & M & 33 & $2 \cdot 23$ & Rh.H.D., E.H., A.S., Minimal A.I., N.S.R., II-C \\
\hline $3 / 12 / 59$ & D. Bre. & 284 & $\mathrm{~F}$ & 29 & $1 \cdot 70$ & Rh.H.D., E.H., M.S., Minimal A.I., N.S.R., I-B \\
\hline $3 / 16 / 59$ & D. Yoh. & 285 & M & 31 & 1.91 & Rh.H.D., E.H., A.S., A.I., M.S., N.S.R., III-C \\
\hline $4 / 27 / 59$ & W. McCa. & 296 & M & 40 & $1 \cdot 67$ & Rh.H.D., E.H., M.S., Minimal A.I., N.S.R., II-C \\
\hline $5 / 4 / 59$ & H. Tal. & 298 & M & 35 & $1 \cdot 82$ & Rh.H.D., E.H., M.S., Minimal A.I., N.S.R., II-C \\
\hline $11 / 16 / 59$ & L. Bal. & 355 & M & 44 & $1 \cdot 74$ & Rh.H.D., E.H., M.S., Minimal A.I., A.F., II-C \\
\hline $12 / 1 / 59$ & L. McCa. & 361 & $\mathrm{~F}$ & 43 & $1 \cdot 69$ & Rh.H.D., E.H., M.S., Minimal A.I., N.S.R., II-C \\
\hline $12 / 7 / 59$ & P. Ose. & 363 & M & 56 & $1 \cdot 79$ & Rh.H.D., E.H., M.S., N.S.R., II-C \\
\hline $3 / 10 / 60$ & W. Mil. & 391 & M & 43 & $1 \cdot 79$ & Rh.H.D., E.H., M.S., Minimal A.I., A.F., II-C \\
\hline \multicolumn{6}{|c|}{$\begin{aligned} \text { Rh.H.D. } & =\text { Rheumatic Heart Disease } \\
\text { E.H. } & =\text { Enlarged Heart } \\
\text { M.S. } & =\text { Mitral Stenosis } \\
\text { M.I. } & =\text { Mitral Insufficiency } \\
\text { A.S. } & =\text { Aortic Stenosis }\end{aligned}$} & $\begin{aligned} \text { A.I. } & =\text { Aortic Insufficiency } \\
\text { T.S. } & =\text { Tricuspid Stenosis } \\
\text { N.S.R. } & =\text { Normal Sinus Rhythm } \\
\text { A.F. } & =\text { Atrial Fibrillation } \\
\text { B.S.A. } & =\text { Body Surface Area }\end{aligned}$ \\
\hline
\end{tabular}

III-C, III-D, II-C, and I-B refer to the physiological and therapeutic cardiac classification of the New York Heart Association.

In all subsequent studies acetylcholine was administered into the right ventricular outflow tract. In study number $211,1.5 \mathrm{mg} . / \mathrm{min}$. was infused. In number $219,3 \mathrm{mg}$. $/ \mathrm{min}$. was infused into the right ventricle. In numbers 251,263 , and $280,2.25 \mathrm{mg} . / \mathrm{min}$. was utilized. In numbers 284 , and $285,4.25 \mathrm{mg}$. $/ \mathrm{min}$. was infused. In numbers $296,298,355,361,363$, and 391 , the infusion rate was $6 \mathrm{mg} . / \mathrm{min}$. All patients were digitalized before cardiac catheterization.

\section{RESULTS}

The data are given in Tables II and III. The data obtained during right heart catheterization alone in these 14 subjects are to be reported separately as part of a similar study in a total of 67 patients (Samet et al., 1961). Acetylcholine infusion during combined heart catheterization resulted in only minimal decreases in pulmonary artery pressure. These changes are not statistically significant (Table III). The changes in left atrial, left ventricular, and systemic arterial pressures and in the diastolic mitral and systolic aortic gradients also are not statistically significant.

In one patient with combined mitral and aortic stenosis, acetylcholine infusion did not alter mean left atrial and left ventricular systolic and diastolic pressure. The mean diastolic left atrial- 
TABLE II

Hamodynamic Data in 14 Patients with Rheumatic Heart Disease (Combined Heart Catheterization)

\begin{tabular}{|c|c|c|c|c|c|c|c|c|}
\hline \multicolumn{2}{|c|}{$\begin{array}{c}\text { Patient } \\
\text { Cath. No. }\end{array}$} & \multirow{2}{*}{$\begin{array}{c}\text { Ventricular } \\
\text { rate/min. } \\
85 \\
87\end{array}$} & \multirow{2}{*}{$\begin{array}{c}\begin{array}{c}\text { Pulmonary } \\
\text { artery } \\
\text { s/d, m }\end{array} \\
56 / 36,43 \\
55 / 34,42\end{array}$} & \multirow{2}{*}{$\begin{array}{c}\begin{array}{c}\text { Brachial } \\
\text { artery } \\
\text { s/d, m }\end{array} \\
112 / 79,89 \\
108 / 75,85\end{array}$} & \multirow{2}{*}{$\begin{array}{c}\begin{array}{c}\text { L.A. } \\
\text { m. }\end{array} \\
28 \\
27\end{array}$} & \multirow{2}{*}{$\begin{array}{c}\begin{array}{c}\text { LA-LV } \\
\text { mean dia- } \\
\text { stolic grad. }\end{array} \\
18 \\
18\end{array}$} & \multirow{2}{*}{$\begin{array}{c}\begin{array}{c}\text { L.V. } \\
\text { s/d }\end{array} \\
106 / 2 \\
106 / 4\end{array}$} & \multirow{2}{*}{$\frac{\begin{array}{c}\text { LV-BA } \\
\text { mean syst- } \\
\text { olic grad. }\end{array}}{-}$} \\
\hline $\begin{array}{l}\text { R. Cos. } \\
198\end{array}$ & $\begin{array}{l}\text { C. } \\
\text { Ac. }\end{array}$ & & & & & & & \\
\hline $\begin{array}{l}\text { M. Win. } \\
211\end{array}$ & $\begin{array}{l}\text { C. } \\
\text { Ac. }\end{array}$ & $\begin{array}{l}84 \\
85\end{array}$ & $\begin{array}{l}49 / 25,33 \\
44 / 25,32\end{array}$ & $\begin{array}{l}112 / 61,78 \\
110 / 60,79\end{array}$ & $\begin{array}{l}16 \\
18\end{array}$ & $\begin{array}{l}16 \\
19\end{array}$ & $\begin{array}{l}102 / 1 \\
100 / 2\end{array}$ & - \\
\hline $\begin{array}{l}\text { C. Bro. } \\
219\end{array}$ & $\begin{array}{l}\text { C. } \\
\text { Ac. }\end{array}$ & $\begin{array}{l}78 \\
66\end{array}$ & $\begin{array}{l}129 / 62,83 \\
125 / 61,79\end{array}$ & $\begin{array}{l}92 / 61,74 \\
55 / 36,48\end{array}$ & $\begin{array}{l}22 \\
25\end{array}$ & $\begin{array}{l}12 \\
12\end{array}$ & $\begin{array}{l}128 / 14 \\
123 / 18\end{array}$ & $\begin{array}{l}33 \\
52\end{array}$ \\
\hline $\begin{array}{l}\text { L. McGow. } \\
251\end{array}$ & $\begin{array}{l}\text { C. } \\
\text { Ac. }\end{array}$ & $\begin{array}{l}92 \\
91\end{array}$ & $\begin{array}{l}42 / 19,25 \\
40 / 19,25\end{array}$ & $\begin{array}{l}93 / 57,71 \\
96 / 59,73\end{array}$ & $\begin{array}{l}17 \\
17\end{array}$ & $\begin{array}{l}13 \\
12\end{array}$ & $\begin{array}{l}84 / 2 \\
88 / 1\end{array}$ & - \\
\hline $\begin{array}{l}\text { B. Ruc. } \\
263\end{array}$ & $\begin{array}{l}\text { C. } \\
\text { Ac. }\end{array}$ & $\begin{array}{l}74 \\
82\end{array}$ & $\begin{array}{l}35 / 19,25 \\
37 / 19,24\end{array}$ & $\begin{array}{l}103 / 57,78 \\
104 / 58,76\end{array}$ & $\begin{array}{l}15 \\
14\end{array}$ & $\begin{array}{l}10 \\
11\end{array}$ & $\begin{array}{r}99 / 6 \\
102 / 6\end{array}$ & - \\
\hline $\begin{array}{l}\text { M. Ham. } \\
280\end{array}$ & $\begin{array}{l}\text { C. } \\
\text { Ac. }\end{array}$ & $\begin{array}{l}76 \\
84\end{array}$ & $\begin{array}{l}30 / 14,19 \\
27 / 13,19\end{array}$ & $\begin{array}{l}125 / 66,89 \\
124 / 66,88\end{array}$ & - & - & $\begin{array}{l}163 / 7 \\
162 / 5\end{array}$ & $\begin{array}{l}40 \\
41\end{array}$ \\
\hline $\begin{array}{l}\text { D. Bre. } \\
284\end{array}$ & $\begin{array}{l}\text { C. } \\
\text { Ac. }\end{array}$ & $\begin{array}{l}84 \\
84\end{array}$ & $\begin{array}{l}19 / 9,14 \\
19 / 9,13\end{array}$ & $\begin{array}{l}113 / 71,88 \\
108 / 69,87\end{array}$ & $\begin{array}{l}2 \\
2\end{array}$ & $\begin{array}{l}5 \\
4\end{array}$ & $\begin{array}{l}107 / 0 \\
105 / 0\end{array}$ & - \\
\hline$\underset{285}{\text { D. Yoh. }}$ & $\begin{array}{l}\text { C. } \\
\text { Ac. }\end{array}$ & $\begin{array}{l}85 \\
85\end{array}$ & $\begin{array}{l}62 / 36,46 \\
64 / 39,48\end{array}$ & $\begin{array}{r}98 / 49,75 \\
101 / 53,75\end{array}$ & $\begin{array}{l}26 \\
28\end{array}$ & $\begin{array}{l}13 \\
11\end{array}$ & $\begin{array}{l}209 / 15 \\
207 / 16\end{array}$ & 二 \\
\hline $\begin{array}{l}\text { W. McCal. } \\
296\end{array}$ & $\begin{array}{l}\text { C. } \\
\text { Ac. }\end{array}$ & $\begin{array}{l}59 \\
55\end{array}$ & $\begin{array}{l}45 / 21,30 \\
47 / 24,33\end{array}$ & - & $\begin{array}{l}16 \\
18\end{array}$ & $\begin{array}{l}12 \\
13\end{array}$ & $\begin{array}{l}98 / 0 \\
93 / 4\end{array}$ & - \\
\hline $\begin{array}{l}\text { H. Tal. } \\
298\end{array}$ & $\begin{array}{l}\text { C. } \\
\text { Ac. }\end{array}$ & $\begin{array}{l}91 \\
82\end{array}$ & $\begin{array}{l}60 / 37,46 \\
52 / 33,39\end{array}$ & $\begin{array}{l}101 / 59,76 \\
102 / 58,72\end{array}$ & $\begin{array}{l}27 \\
26\end{array}$ & $\begin{array}{l}21 \\
22\end{array}$ & $\begin{array}{l}106 / 1 \\
109 / 2\end{array}$ & $\begin{array}{l}17 \\
17\end{array}$ \\
\hline $\begin{array}{l}\text { L. Bal. } \\
355\end{array}$ & $\begin{array}{l}\text { C. } \\
\text { Ac. }\end{array}$ & $\begin{array}{l}87 \\
90\end{array}$ & $\begin{array}{l}41 / 23,29 \\
39 / 22,29\end{array}$ & - & $\begin{array}{l}14 \\
15\end{array}$ & $\begin{array}{l}8 \\
8\end{array}$ & $\begin{array}{l}115 / 0 \\
114 / 1\end{array}$ & $=$ \\
\hline $\begin{array}{l}\text { L. McCan. } \\
361\end{array}$ & $\begin{array}{l}\text { C. } \\
\text { Ac. }\end{array}$ & $\begin{array}{l}87 \\
88\end{array}$ & $\begin{array}{l}29 / 14,21 \\
27 / 15,21\end{array}$ & $\begin{array}{l}124 / 74,96 \\
122 / 72,94\end{array}$ & $\begin{array}{l}12 \\
11\end{array}$ & $\begin{array}{l}13 \\
13\end{array}$ & $\begin{array}{l}120 / 0 \\
117 / 0\end{array}$ & - \\
\hline $\begin{array}{l}\text { P. Ose. } \\
363\end{array}$ & $\begin{array}{l}\text { C. } \\
\text { Ac. }\end{array}$ & $\begin{array}{l}83 \\
79\end{array}$ & $\begin{array}{l}38 / 11,22 \\
36 / 8,18\end{array}$ & $\begin{array}{l}130 / 71,96 \\
123 / 67,91\end{array}$ & $\begin{array}{l}8 \\
8\end{array}$ & $\begin{array}{l}3 \\
3\end{array}$ & $\begin{array}{l}113 / 11 \\
112 / 11\end{array}$ & 二 \\
\hline $\begin{array}{l}\text { W. Mil. } \\
391\end{array}$ & $\begin{array}{l}\text { C. } \\
\text { Ac. }\end{array}$ & $\begin{array}{l}73 \\
71\end{array}$ & $\begin{array}{l}33 / 22,25 \\
30 / 21,24\end{array}$ & $\begin{array}{l}125 / 77,90 \\
124 / 75,92\end{array}$ & $\begin{array}{l}13 \\
13\end{array}$ & $\begin{array}{l}6 \\
7\end{array}$ & $\begin{array}{l}118 / 9 \\
116 / 6\end{array}$ & - \\
\hline Mean & $\begin{array}{l}\text { C. } \\
\text { Ac. }\end{array}$ & $\begin{array}{l}81 \\
81\end{array}$ & $\begin{array}{l}48 / 25,33 \\
46 / 24,32\end{array}$ & $\begin{array}{l}111 / 65,83 \\
106 / 62,80\end{array}$ & $\begin{array}{l}17 \\
17\end{array}$ & $\begin{array}{l}12 \\
12\end{array}$ & $\begin{array}{l}119 / 5 \\
118 / 5\end{array}$ & $\begin{array}{l}30 \\
36\end{array}$ \\
\hline
\end{tabular}

left ventricular gradient was therefore unchanged. However brachial artery pressure fell with a resultant increase in the mean systolic left ventricular to brachial artery gradient from 33 to $52 \mathrm{~mm} . \mathrm{Hg}$ (Fig. 1 and 2).

\section{Discussion}

The data provided in the present study demonstrate directly for the first time that right heart intracardiac acetylcholine infusion does not alter mean left atrial pressure. Wood et al. (1957) and Söderholm and Werkö (1959) measured pulmonary arterial wedge pressures in patients with mitral stenosis during acetylcholine infusion but direct left atrial pressure data was not obtained.

Since left heart pressures and gradients in subjects with rheumatic heart disease are not altered 
TABLE III

Hemodynamic Data: Significance of Changes in 14 Patients with Rheumatic Heart Disease

\begin{tabular}{|c|c|c|c|c|c|c|}
\hline & \multicolumn{3}{|c|}{ Pulmonary artery } & \multicolumn{3}{|c|}{ Brachial artery } \\
\hline & $\mathrm{s}$ & $\mathrm{d}$ & $\mathrm{m}$ & s & $\mathrm{d}$ & $\mathrm{m}$ \\
\hline I. All patients. Mean control & $47 \cdot 7$ & $24 \cdot 8$ & $32 \cdot 9$ & $110 \cdot 7$ & $65 \cdot 2$ & $83 \cdot 4$ \\
\hline Mean acetylcholine & $45 \cdot 8$ & $24 \cdot 4$ & $31 \cdot 8$ & $106 \cdot 4$ & $62 \cdot 3$ & $80 \cdot 0$ \\
\hline $\mathrm{N}=14$. Mean change & $-1 \cdot 8$ & $-0 \cdot 4$ & $-1 \cdot 0$ & $-4 \cdot 3$ & $-2 \cdot 0$ & $-3 \cdot 4$ \\
\hline Significance of change & $0.05>P>0.02$ & $0.4>P>0.3$ & $0 \cdot 2>\mathrm{P}>0 \cdot 1$ & $0 \cdot 2>\mathrm{P}>0 \cdot 1$ & $0.3>P>0.2$ & $0 \cdot 2>P>0 \cdot 1$ \\
\hline
\end{tabular}

\begin{tabular}{|c|c|c|c|c|c|c|}
\hline & Vent. rate & L.A. mean & $\begin{array}{l}\text { L.A.-L.V. } \\
\text { mean } \\
\text { gradient }\end{array}$ & $\begin{array}{c}\text { L.V. } \\
\text { Systolic }\end{array}$ & $\begin{array}{l}\text { L.V. } \\
\text { Diastolic }\end{array}$ & $\begin{array}{l}\text { L.V.-B.A. } \\
\text { mean } \\
\text { gradient }\end{array}$ \\
\hline All patients. Mean control & $81 \cdot 3$ & $16 \cdot 61$ & $11 \cdot 5$ & $119 \cdot 14$ & $4 \cdot 9$ & $30 \cdot 0$ \\
\hline Mean acetylcholine & $80 \cdot 6$ & $17 \cdot 1$ & $11 \cdot 7$ & $118 \cdot 14$ & $5 \cdot 4$ & $36 \cdot 7$ \\
\hline$N=14$. Mean change & 0.64 & $+0 \cdot 6$ & $+0 \cdot 2$ & $-1 \cdot 0$ & $+0 \cdot 5$ & $+6 \cdot 7$ \\
\hline Significanc. of change & $0.7>P>0.6$ & $0.3>P>0.2$ & $0.6>P>0.5$ & $0 \cdot 2>P>0 \cdot 1$ & $0.3>P>0 \cdot 2$ & $0 \cdot 4>P>0 \cdot 3$ \\
\hline
\end{tabular}

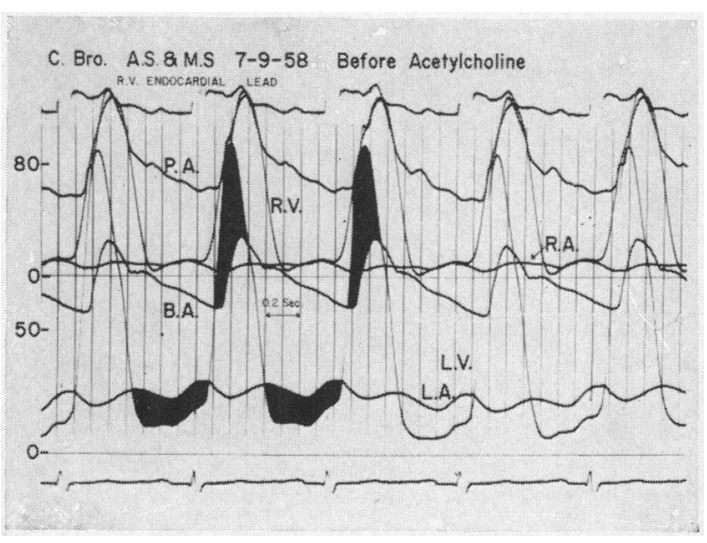

FIG. 1.-Right and left heart pressures in C. Bro., a patient with combined mitral and aortic stenosis. Control pressures prone, prior to acetylcholine infusion.

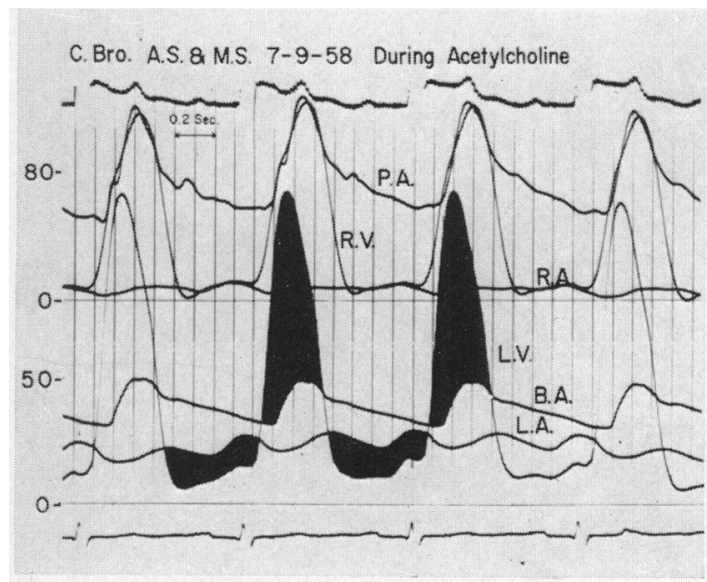

FIG. 2.-Acetylcholine infusion results in systemic arterial hypotension and an increase in the mean systolic left ventricular brachial artery gradient from 33 to $52 \mathrm{~mm}$. $\mathrm{Hg}$.

by right heart acetylcholine infusion, and since heart rate and cardiac output are only minimally affected by acetylcholine (Samet et al., 1961), and since central blood volume remains unchanged in the hypoxic normal subject after acetylcholine infusion (Fritts et al., 1958), a decrease in pulmonary artery pressure after acetylcholine infusion could be interpreted as pulmonary artery vasodilation. The minimal fall in pulmonary arterial pressures noted after drug infusion in the present study again demonstrates the limited clinical value of acetylcholine as a pulmonary vasodilator agent. 


\section{SUMMARY}

Right heart acetylcholine infusion was employed in the course of combined right and leftheart catheterization in 14 patients with rheumatic heart disease. Left atrial mean pressure and the mean diastolic mitral gradient and the mean systolic aortic gradient were not altered by acetylcholine infusion. Pulmonary arterial pressure fell only slightly after acetylcholine infusion. Acetylcholine offers little promise as a clinically useful pulmonary vasodilator agent.

\section{REFERENCES}

Bernstein, W. H., Fierer, E. M., Laszlo, M. H., Samet, P., and Litwak, R. S. (1960). Brit. Heart J., $22,37$. Fritts, H. W., Jr., and Cournand, A. (1959). Pulmonary Circulation. Grune and Stratton Inc., New York. -, Harris, P., Clauss, R. H., Odell, J. E., and Cournand, A. (1958). J. clin. Invest., 37, 99.

Samet, P., Litwak, R. S., Bernstein, W. H., Fierer, E. M., and Silverman, L. M. (1959). Circulation, $19,517$. - Bernstein, W. H., Fernandez, L., and De Victoria, W. (1961). Amer. J. Cardiol. In press. Söderholm, B., and Werkö, L. (1959). Brit. Heart J., 21, 1.

Wood, P., Besterman, E. M., Towers, M. K., and McIlroy, M. B. (1957). Brit. Heart J., 19, 279. 